Check for updates

Cite this: RSC Adv., 2018, 8, 30701

Received 4th June 2018

Accepted 6th August 2018

DOI: $10.1039 / \mathrm{c} 8 \mathrm{ra0} 4765 \mathrm{k}$

rsc.li/rsc-advances

\section{Current density distribution and optimization of the collection electrodes of a honeycomb wet electrostatic precipitator}

\author{
Chenghang Zheng, Yifan Wang, Xuefeng Zhang, Zhengda Yang, Shaojun Liu, \\ Yishan Guo, Yongxin Zhang, Yi Wang and Xiang Gao (D)*
}

\begin{abstract}
Wet electrostatic precipitators (ESPs) demonstrate a robust adaptability for particulate matter control and have been confirmed to be a promising technology for removing particles and sulfuric acid aerosol from flue gas. Recent studies have shown that removing fine particles or sulfuric acid aerosol from wet ESPs requires further development. Among the components of wet ESPs, discharge electrode configurations are crucial for determining the performance of wet ESPs. This paper reports the corona discharge characteristics and removal performance of sulfuric acid aerosol using different discharge electrode configurations in a honeycomb wet ESP experimental system. Two key parameters, namely, $V-I$ characteristics and current density distribution, with different discharge electrode geometries (e.g., electrode type, spike spacing, and spike length) and rotation angles, were investigated by using a novel electrical parameter measurement system to evaluate the effects of these parameters on corona discharge. The results showed that triple-spike and sawtooth electrodes exhibit the highest average current density. The average current density of the triple-spike electrode increased with the spike length from $10 \mathrm{~mm}$ to $20 \mathrm{~mm}$, and the peak current density distribution on the collection electrode increased by $62.1 \%$, but the current density decreased sharply away from the spike. Moreover, the average peak current density decreased by $30.1 \%$ when the spike spacing was $25 \mathrm{~mm}$ given the sharp point discharge suppression when spikes were significantly dense. The electrode configuration was optimized on the basis of the current density distribution. The highest removal efficiency of sulfuric acid aerosol was $99.2 \%$ at a specific collection area of $23.09 \mathrm{~m}^{2}\left(\mathrm{~m}^{3} \mathrm{~s}^{-1}\right)^{-1}$.
\end{abstract}

\section{Introduction}

Particulate matter (PM) air pollution is a well-recognized carcinogen for humans and has caused global concerns over the past few years. ${ }^{1}$ The World Health Organization contends that exposure to PM may cause 3.7 million premature deaths worldwide. ${ }^{2}$ Long-term exposure to PM is related to morbidity and mortality due to lung cancer. ${ }^{3-5}$ Fine and ultrafine particles are extensively hazardous to human health. These particles can damage the respiratory system and reach the deepest regions of the lungs and even the cardiovascular system when inhaled. ${ }^{6-8}$ The control of particulate emissions from the original sources is an efficient method for clean production.

Some sulfur in fuel can be oxidized to $\mathrm{SO}_{3}$ during the combustion process in a boiler. The $\mathrm{SO}_{3}$ concentration increases significantly after selective catalytic reduction because $0.25-1.25 \%$ of $\mathrm{SO}_{2}$ can be oxidized to $\mathrm{SO}_{3} .{ }^{9}$ The flue gas

State Key Lab of Clean Energy Utilization, State Environmental Protection Engineering Center for Coal-Fired Air Pollution Control, Institute for Thermal Power Engineering, Zhejiang University, 38 Zheda Road, Hangzhou 310027, People's Republic of China. E-mail:xgao1@zju.edu.cn; Fax: +86-571-87951616; Tel: +86-571-87951335 temperature can be quenched to below $323.15 \mathrm{~K}$, and the corresponding moist gas is saturated when flue gas enters the wet flue gas desulfurization (WFGD) system. ${ }^{\mathbf{1 0 , 1 1}}$ Sulfuric acid aerosol is formed by combining $\mathrm{SO}_{3}$ and flue gas moisture, and the typical size of sulfuric acid aerosol is approximately $0.1 \mu \mathrm{m} .{ }^{\mathbf{1 2}}$

Wet ESPs demonstrate a robust adaptability for PM control, especially for particulates that are corrosive, sticky, and exhibit relatively high or low resistivity. Back corona discharge and dust re-entrainment can be avoided because the dust cake on the collection electrode is removed by water film efficiently. ${ }^{13}$ Wet ESPs are typically installed downstream of the WFGD system to remove fine dust and sulfuric acid aerosol from flue gas. ${ }^{\mathbf{1 4 , 1 5}}$ However, removing fine particles or sulfuric acid aerosol using wet ESPs requires further development given poor charging capacity, and the particle removal efficiency of wet ESPs decline further with the increase in particle concentration. ${ }^{9,12,16}$

Discharge current density and distribution are major parameters of operating performance. The distribution of electric field bears a solid relationship with an electrode. ${ }^{17} \mathrm{~A}$ high discharge current density leads to effective particle charging, and the charged particles provide high migration velocity and removal efficiency. ${ }^{18}$ The removal efficiency of an 
ESP can be improved by increasing the uniformity of the discharge current density distribution. ${ }^{19}$ ESPs are classified as cylindrical and plate types on the basis of the shape of the collection electrodes. ${ }^{\mathbf{1 3 2 0}}$ Numerous studies on the discharge characteristics of wire-plate ESPs have been reported; these studies include investigations of emitter geometry and material on the electrode corona discharge to optimize the discharge electrode through experimental and numerical methods. ${ }^{21-25}$ Electrodes with different shapes, diameters, and intervals were tested in an experimental-scale ESP at high temperatures, ${ }^{26,27}$ and migration velocity was also investigated. ${ }^{28,29}$ Corona discharge and particle collection characteristics in a wet ESP with a fine water mist were discussed..$^{23030}$ The electric power consumption of the ESP with spikes on both sides is nearly twice as much as that of the ESP with spikes on only one side. ${ }^{31}$ The electrical characteristics and ionic wind vary with the spike point spacing. ${ }^{32}$ A numerical model of ESP has been developed to describe the electric field, and the rotation of the discharge electrode optimizes its electric field. ${ }^{33}$ Several researchers have investigated the discharge and removal characteristics of a wirecylinder ESP. ${ }^{34,35}$ Moreover, a novel electrode that contains rare earth materials has been presented and investigated. ${ }^{36}$ In practical applications, barbed discharge and honeycomb collection electrodes are widely used in wet ESPs. However, the influence of discharge electrode configuration on corona discharge has not been investigated systematically, and minimal work has been conducted to reveal the correlation between current density distribution and optimization of wet ESP, especially in terms of honeycomb wet ESP.

In this study, a honeycomb wet ESP experimental system was designed and operated with different discharge electrode configurations. The discharge characteristics of wet ESP were evaluated by using the $V-I$ characteristic and current density distribution. Influences of discharge electrode type, spike length $L$, spike spacing $S$, and rotation angle $\alpha$ of the discharge electrode on the discharge characteristics of wet ESP were investigated and discussed. The removal efficiency of sulfuric acid aerosol in wet ESP was analyzed to determine the effects of optimizing current density distribution to enhance wet ESP. Conclusions drawn from this study could provide a basis for wet ESP design and application.

\section{Experimental setup and methods}

A schematic of the experimental system is illustrated in Fig. 1. The system consisted of five parts, namely, (1) a sulfuric acid aerosol generating system, (2) a laboratory-scale wet ESP, (3) a negative DC power supply system, (4) a current parameter measurement system, and (5) a sulfuric acid aerosol sampling device.

\subsection{Experimental setup}

A wire-honeycomb negative DC corona discharge configuration consisted of a wire discharge electrode and a honeycomb collection electrode. The honeycomb collection electrode was a vertical and hexagonal tube with a single pipe $(50 \mathrm{~mm}$ in circumradius and $1000 \mathrm{~mm}$ in height), and the collection electrode was grounded. Different geometry electrodes with a diameter of $10 \mathrm{~mm}$ and a length of $1000 \mathrm{~mm}$ were used as discharge electrodes. An adjustable DC negative voltage from $0 \mathrm{kV}$ to $40 \mathrm{kV}$ and a maximum current of $10 \mathrm{~mA}$ were generated by a high-voltage power supply. A negative high voltage was applied to the discharge electrode. Simulated flue gas was generated by an air fan and then heated by an electric heater. In the present study, the flue gas was humidified using a humidifying device to ensure saturation. $\mathrm{SO}_{3}$ generated by an $\mathrm{SO}_{3}$ generator was injected into the flue gas and combined with $\mathrm{H}_{2} \mathrm{O}$ to form sulfuric acid aerosol under this condition. The simulated flue gas entered the bottom of the wet ESP, and ultrafine particles (sulfuric acid aerosol) were charged and captured. A sulfuric acid aerosol sampling device includes a sampling port, a diluter (Dekati Ltd., Finland), an electrical low-pressure impactor (ELPI ${ }^{+}$; Dekati Ltd., Finland), and a vacuum pump. The gas containing sulfuric acid aerosol was diluted eight times by using the diluter. The concentration of the sulfuric acid aerosol in the flue gas was measured online by using ELPI ${ }^{+}$.

For the experiments, four types of barbed discharge electrodes (single-spike, double-spike, triple-spike, and sawtooth) and a smooth wire electrode were used, as depicted in Fig. 1. The spikes of barbed electrodes were rotated at $60^{\circ}$ per row to ensure discharge uniformity. Three types of barbed electrodes can be further subdivided in accordance with $L(10,15$, and 20 $\mathrm{mm})$ and $S(25,50,100,150$, and $200 \mathrm{~mm})$, where $L$ and $S$ are the spike spacing and length of the electrode, respectively. The sawtooth electrode had 30 sharp points per row, a disk spacing of $50 \mathrm{~mm}$, an inside diameter of $15 \mathrm{~mm}$, an outside diameter of $20 \mathrm{~mm}$, and a thickness of $1 \mathrm{~mm}$. In this experiment, a comparative analysis of the electrical characteristics of wet ESP in aspects of discharge current density and distribution were investigated using 23 kinds of discharge electrodes.

\subsection{Electrical parameter measurement system}

A novel probe and a current measurement system for the experiments were designed to measure the current density distribution on the collection electrode, as demonstrated in Fig. 2. An insulation ring was connected to a metal shell, and then a $7 \mathrm{~mm}$-diameter probe was embedded in the ring. The interval between the metal shell and the probe prevented a short circuit caused by the condensation of sulfuric acid aerosol on the collection electrode surface. The integrated probes were fixed on the collection electrode. Probes were electrically isolated by the ring from the rest area of the collection electrode. To ensure the accuracy of the current density measurement and minimize the influence of probes on the local electric field, probes were connected to the microammeter and adjusted by an anchor nut to maintain a flat surface with the collection electrode.

The current measurement system could automatically scan and record the current of each probe on the collection electrode. Timing relays, circuit boards, current collecting devices, and a digital micro-ammeter with an accuracy of $10^{-6}$ A were used in the system. Nine probes were selected in each row, and probes 


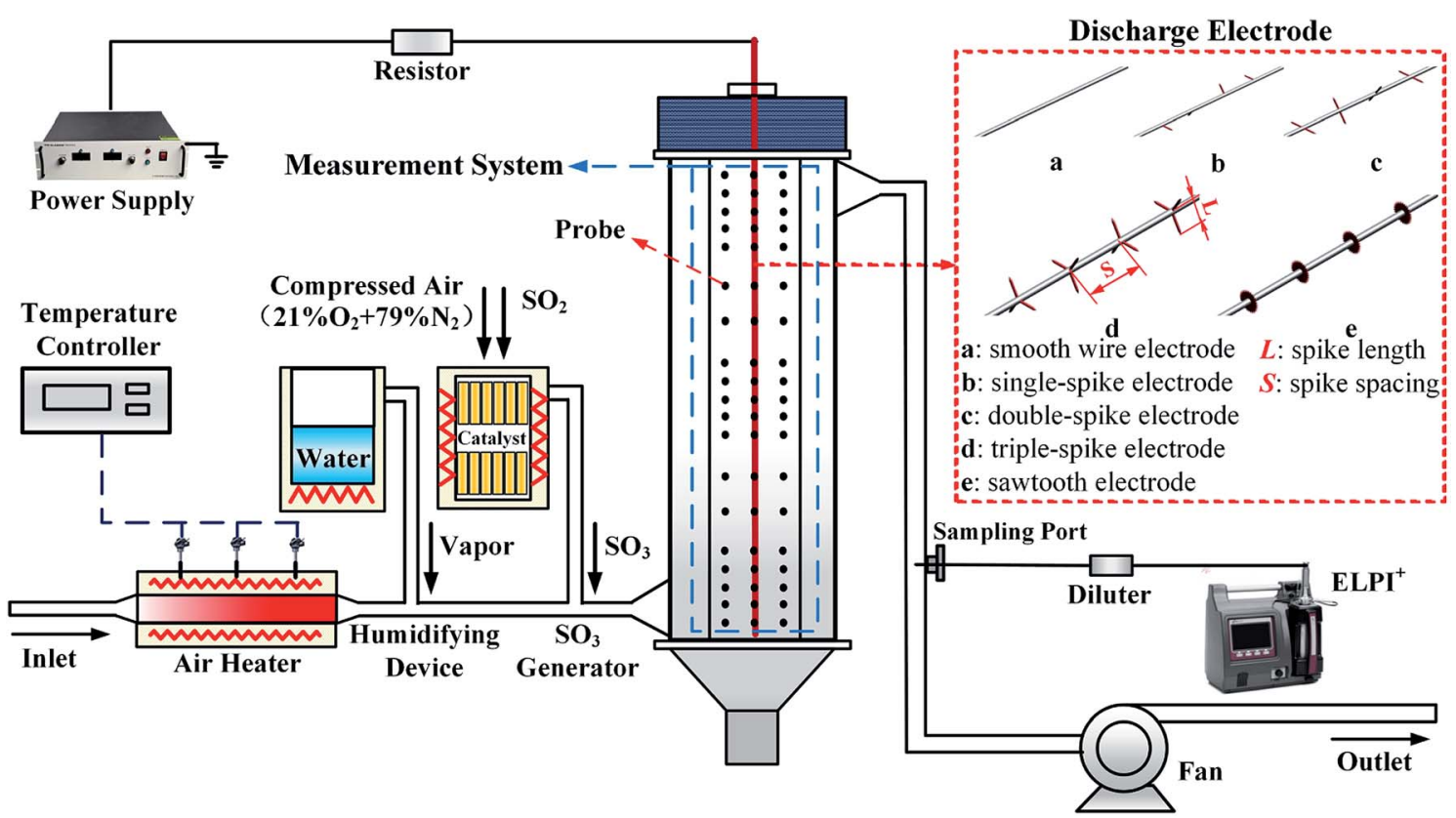

Fig. 1 Schematic of the experimental system.

were encrypted at the ends and center of the wet ESP to ensure the integrity of the measurement results. A total of 19 rows were found on the direction of gas flow with an interval of $25 \mathrm{~mm}$ or $100 \mathrm{~mm}$, and 171 probes were fixed on the surface of the collection electrode. The measuring circuit was grounded after connecting the micro-ammeter, and the bypass was grounded directly. The relay was controlled by software, and the other probes were connected to the bypass to ensure that only one probe was connected to the measuring circuit. In addition, the data of the micro-ammeter was transmitted to and recorded in the current collecting device. The switching period was selected as $3 \mathrm{~s}$, and the data of each probe was recorded at $2.7 \mathrm{~s}$ before switching to the next probe to avoid the influence of the induced current generated by the relay during the switching. Each testing electrode configuration consumed $513 \mathrm{~s}$. This system provided a real-time monitoring of the current density distribution on the collection electrode and improved measurement efficiency and accuracy significantly. The applied voltage and discharge current could be acquired directly from the display panel of the high-voltage power supply.

\subsection{Experimental approach}

Different parameters, such as electrode type, configuration (e.g., spike length $L$, spike spacing $S$, and rotation angle $\alpha$ of the electrode), and voltage/current, were varied and focused on investigating the discharge characteristics of the wet ESP. A corona onset voltage was determined as the voltage where the corona began. The discharge current that corresponds to the applied voltage was detected after the corona onset. The current was nearly constant when the corona discharge stabilized. A spark voltage was determined as the voltage that continuously
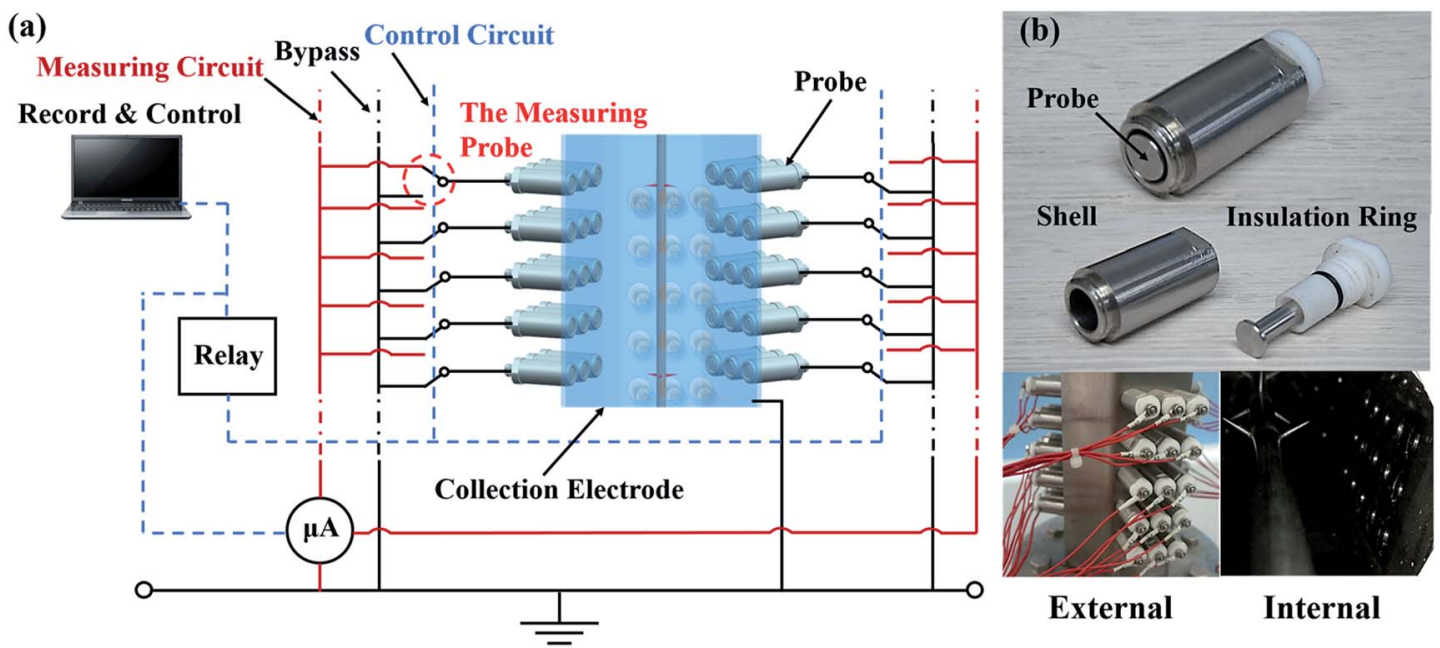

Fig. 2 Current measurement system: (a) schematic and (b) probe. 
sparked, and a leap in the secondary current was observed. Consequently, the applied voltage decreased.

The high-voltage power supply was switched on when the discharge electrode reached the set configuration. In the current density distribution experiments, the detailed current density distribution on the collection electrode was recorded, and the current density was calculated when the applied voltage or discharge current reached the set point.

For this experiment, the current density of probe $J_{\mathrm{p}}$ was calculated using eqn (1).

$$
J_{\mathrm{p}}=\frac{4 i_{\mathrm{p}}}{\pi d^{2}},
$$

where $J_{\mathrm{p}}$ is the current density of probe; $i_{\mathrm{p}}$ is the current of the probe, which is recorded by the current collecting devices; and $d$ is the diameter of the probe-measuring surface, which is $7 \mathrm{~mm}$ in the experiment.

The removal efficiency of sulfuric acid aerosol was calculated using eqn (2), in which the sulfuric acid aerosol concentration was measured by using ELPI ${ }^{+}$.

$$
\eta(\%)=\frac{\sum c_{\text {off }}\left(r_{\mathrm{i}}\right)-\sum c_{\mathrm{on}}\left(r_{\mathrm{i}}\right)}{\sum c_{\text {off }}\left(r_{\mathrm{i}}\right)} \times 100 \%
$$

where $\eta$ is the removal efficiency of sulfuric acid aerosol; $c_{\text {off }}\left(r_{\mathrm{i}}\right)$ is the fractional concentration without corona discharge, $\mathrm{mg}$ $\mathrm{m}^{-3}$; and $c_{\mathrm{on}}\left(r_{\mathrm{i}}\right)$ is the fractional concentration with corona discharge, $\mathrm{mg} \mathrm{m}^{-3}$.

\section{Results and discussion}

\subsection{Discharge characteristics affected by discharge electrode geometry}

3.1.1. Effect of discharge electrode type. Fig. 3 exhibits the $V-I$ characteristics of wet ESP with different discharge electrode types. The barbed discharge electrodes (e.g., single-spike, double-spike, triple-spike, and sawtooth) and a smooth wire electrode were used as the discharge electrode. $L$ was $20 \mathrm{~mm}$, and $S$ was $50 \mathrm{~mm}$. An average current density refers to the current density distribution on the collection electrode with a $0.3 \mathrm{~m}^{2}$ area. Average current density was determined by the discharge electrode type. The corona onset voltage of the smooth wire electrode was $23.9 \mathrm{kV}$, which was 2.5 to 3.8 times higher than the other electrodes, and spark discharge appeared when the average current density only reached $0.233 \mathrm{~mA} \mathrm{~m}^{-2}$. The average current density was higher in the barbed electrode than in the smooth wire electrode at the same applied voltage. The average current density increased with the applied voltage when the corona onset voltage was exceeded, and the slope of the $V-I$ characteristic curve and the number of sharp points had a positive correlation. For the barbed electrodes, sawtooth had the maximum sharp points. Triple-spike, double-spike, and single-spike electrodes were sorted in a descending order on the basis of the number of sharp points. The spark discharge current density and the number of sharp points also had a positive correlation. Spark discharge appeared in the singlespike electrode when the average current density reached $16.67 \mathrm{~mA} \mathrm{~m}^{-2}$. The sawtooth electrode still maintained a stable

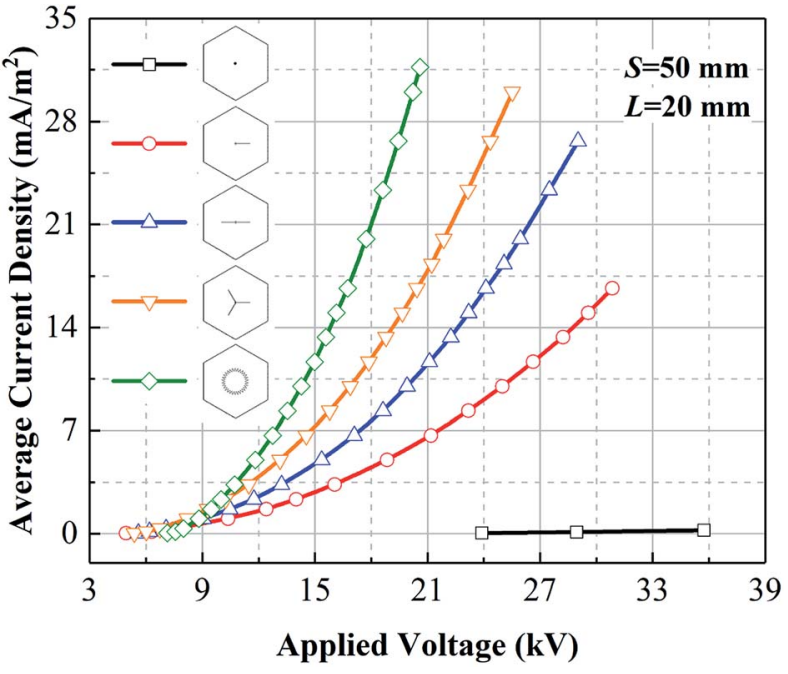

Fig. 3 Effect of the discharge electrode type on the $V-$ I characteristics.

corona discharge when the average current density was 33.33 $\mathrm{mA} \mathrm{m}{ }^{-2}$. The corona onset voltage was $2-3 \mathrm{kV}$ higher in the sawtooth electrode than other barbed electrodes. This phenomenon might be due to the difference of the electric field on the sharp points. Typically, the corona discharge current is emitted from sharp points, namely, "emitters", on the discharge electrode. The sharp point curvature radius was smaller in the triple-spike electrode than in the sawtooth electrode. Thus, the corona onset electric field intensity was higher in the triplespike electrode than in the sawtooth electrode, as expressed in eqn (3). The sawtooth discharge electrode required a high voltage to produce a stable corona discharge, and its corona onset voltage was high. The corona discharge easily occurred when the curvature radius of the sharp point was small. More ions were released from the sawtooth electrode than from the triple-spike electrode at the same voltage after the corona onset because of added sharp points. The average current density of the sawtooth electrode exceeded that of the triple-spike electrode rapidly.

The corona onset electric field intensity on the sharp point was determined by Peek's law as follows:

$$
E_{\mathrm{S}}=E_{0} m\left(\delta+0.0308 \sqrt{\frac{\delta}{r_{0}}}\right),
$$

where $E_{\mathrm{S}}$ is the corona onset electric field intensity on the sharp point surface, $E_{0}$ is a constant at $3.1 \times 10^{6} \mathrm{~V} \mathrm{~m}^{-1}, \mathrm{~m}$ is the dimensionless surface parameter, $r_{0}$ is the curvature radius of the sharp point, and $\delta$ is the relative density of gas with respect to the normal condition of $273.15 \mathrm{~K}$.

$$
\delta=\frac{T_{\mathrm{S}} P}{P_{\mathrm{S}} T},
$$

where $T_{\mathrm{S}}$ and $T$ represent $273.15 \mathrm{~K}$ and the local temperature, respectively; and $P_{\mathrm{S}}$ and $P$ correspond to $101325 \mathrm{~Pa}$ and the local pressure.

Fig. 4 displays the current density distribution on the collection electrode of the different barbed electrodes. Nearly all 


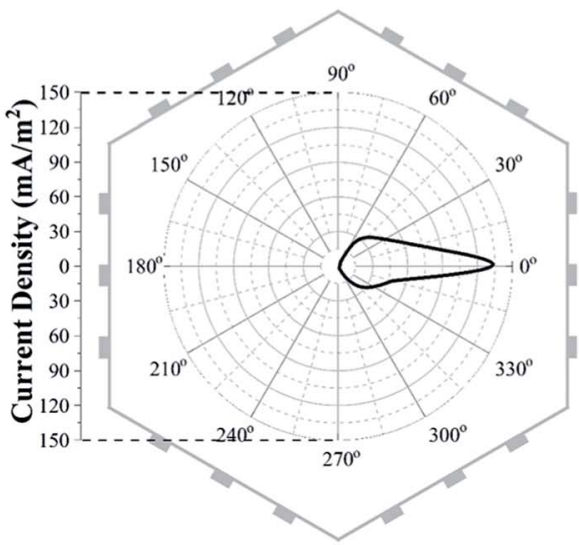

(a) Single-spike Electrode

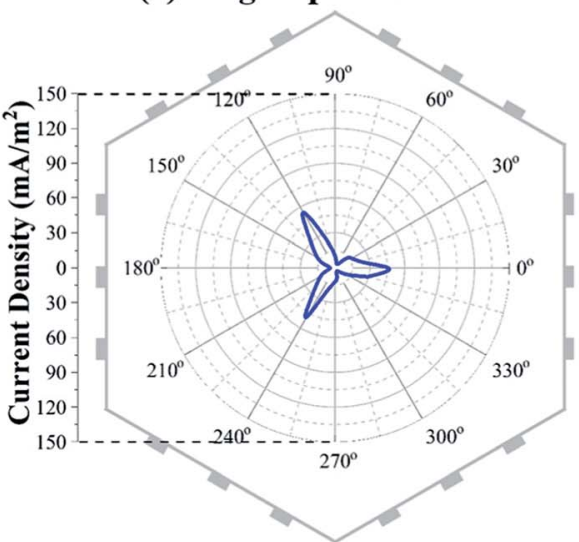

(c) Triple-spike Electrode

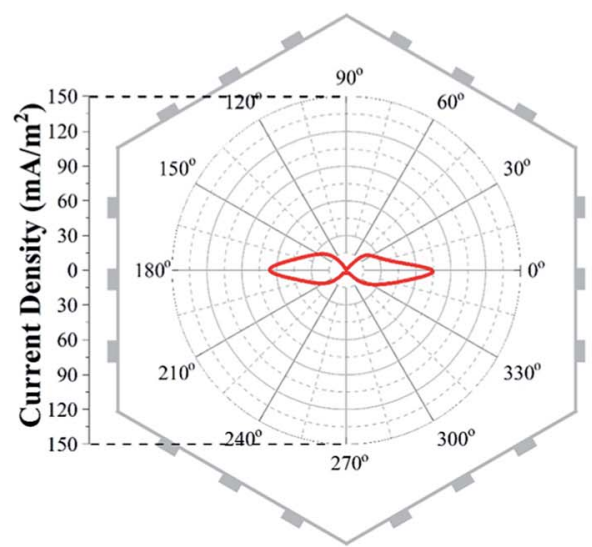

(b) Double-spike Electrode

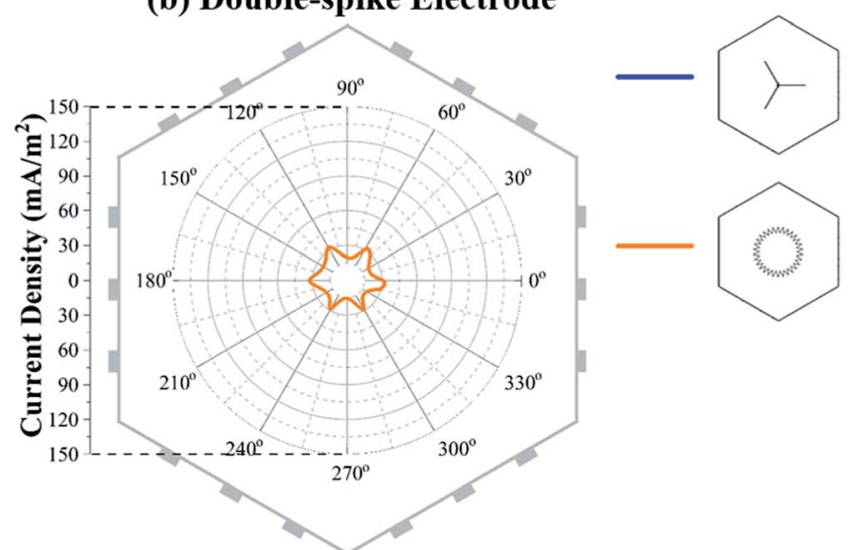

(d) Sawtooth Electrode
Discharge current $=3 \mathrm{~mA}$

$S=50 \mathrm{~mm}, L=\mathbf{2 0} \mathrm{mm}$

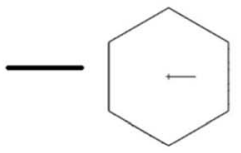

$\longrightarrow$

Fig. 4 Current density distribution on the collection electrode of different barbed electrodes.

the measuring results of the probes were zero given the weak average current density of a smooth wire electrode. Thus, its current density distribution was not shown in this figure. $L$ was $20 \mathrm{~mm}, S$ was $50 \mathrm{~mm}$, and the discharge current was $3 \mathrm{~mA}$. The length on the polar axis refers to the current density of the probe with the area of $3.85 \times 10^{-5} \mathrm{~m}^{2}$. The current density distribution on the collection electrode was determined on the basis of the discharge electrode type. The current density distribution of single-spike, double-spike, and triple-spike electrodes was similar to the geometry of the electrode. The current density distribution on the collection electrode that faces the spikes was the largest and sharply decreased along the two sides of the spikes. The peak current densities were 60.5\%, $133.1 \%$, and $295.8 \%$ higher in the single-spike electrode $(I=3 \mathrm{~mA})$ than in the double-spike, triple-spike, and sawtooth electrodes, respectively. However, its current density distribution was significantly uneven. The current density of a two-thirds area on the collection electrode was zero. Spikes played a dominant part in the barbed electrode discharge. Peak current density decreased with the increase in spikes per row, but the current density distribution was uniform. The current density distribution of the sawtooth electrode on the collection electrode was hexagonal and relatively uniform, and all its current density exceeded zero. The current densities varied with the distance between the sawtooth and collection electrodes. The largest and smallest current densities were positioned closest and farthest to the sawtooth electrode, respectively.

3.1.2. Effect of spike length. Fig. 5 illustrates the $V-I$ characteristics of the wet ESP with various spike lengths and different discharge electrode types. Single-spike, double-spike, and triple-spike electrodes were used as the discharge electrodes. $L$ was 10,15 , and $20 \mathrm{~mm}$, and $S$ was $50 \mathrm{~mm}$. Fig. 5 depicts that the average current density increased with the spike length, but the spark voltage decreased. Owing to these factors, the maximum current density at the spark voltage was enhanced. The effect of the increase in spike length on corona discharge was relatively less effective than the increase in spikes per row.

Fig. 6(a) demonstrates the current density distribution on the collection electrode of different triple-spike electrodes with various spike lengths $(10,15$, and $20 \mathrm{~mm}) . S$ was $50 \mathrm{~mm}$, and the discharge current was $3 \mathrm{~mA}$. Fig. 6(a) displays that the peak current density that faces the spikes increased with the spike length, but the current density distribution on both sides of the spikes decreased. The current density distribution on the collection electrode gradually shrank with the increase in spike length. The peak current density of the triple-spike electrodes increased by $62.1 \%$ with the increase in spike length from $10 \mathrm{~mm}$ to $20 \mathrm{~mm}$, but the current density at the angular bisector of adjacent spikes gradually decreased to zero. The peak current 


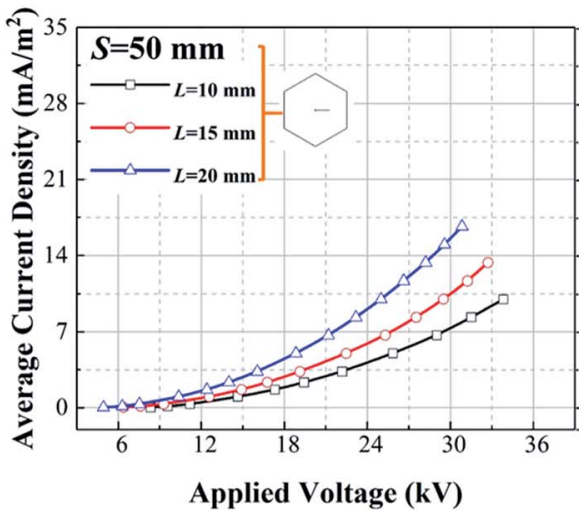

(a) Single-spike Electrode

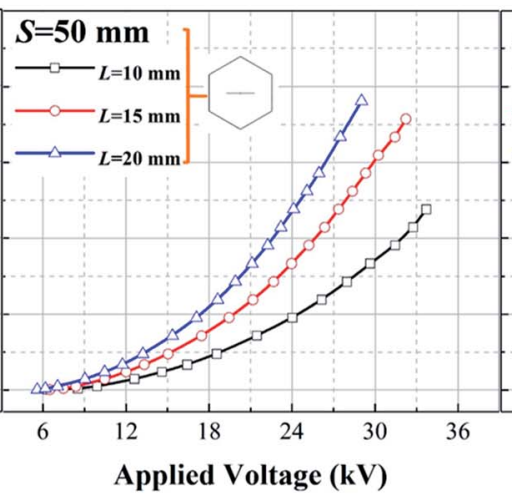

(b) Double-spike Electrode

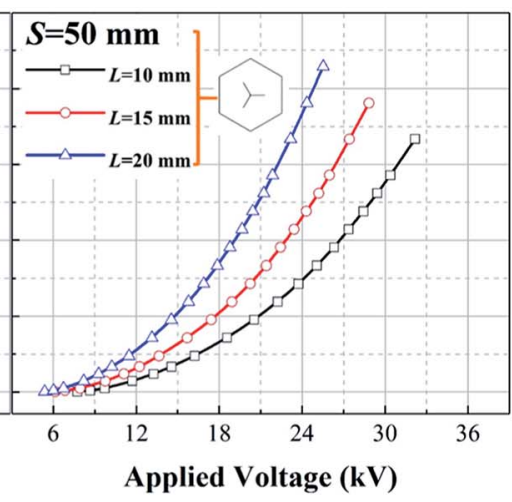

(c) Triple-spike Electrode

Fig. 5 Effect of spike length on the $V-/$ characteristics.

densities of the double-spike and single-spike electrodes increased by $63.7 \%$ and $68.3 \%$, correspondingly.

This phenomenon may be due to the difference in the ion diffusion region in wet ESP. The average current density increased with the spike length, but the ion diffusion region generated by the sharp point corona discharge shrank gradually. That is, the area on the collection electrode that cannot be reached by ions increased and formed a low-ion charge density area.

In this study, four positions were selected in accordance with the symmetry of the triple-spike and collection electrodes, especially for the current density distribution analysis, to clearly demonstrate the effect of the spike length on discharge characteristics. Fig. 6(b) presents that the trend of the current density change in four positions was different with the increase in the spike length from $10 \mathrm{~mm}$ to $20 \mathrm{~mm}$. The current density of Position no. 1 was significantly affected by the spike length. The current density of Position no. 2 initially increased and then decreased. The current density of Position no. 3 and 4 decreased with the increase in the spike length. Moreover, the current density of Position no. 4 dropped to zero when the spike length was $20 \mathrm{~mm}$.
3.1.3. Effect of spike spacing. Spike spacing significantly affects corona discharge. In this experiment, five triple-spike electrodes with various spike spacings (i.e., 25, 50, 100, 150, and $200 \mathrm{~mm}$ ) were used as the discharge electrodes, and $L$ was $20 \mathrm{~mm}$. The relation of spike spacing to the total of spikes is presented in Table 1. Fig. 7 depicts that the average current density increased with the decrease in spike spacing. In addition, the spark discharge current density increased with the decrease in spike spacing, but the spark voltage was nearly unchanged. Triple-spike electrodes, with spike spacings of 25 and $50 \mathrm{~mm}$, maintained a stable corona discharge when the average current density was $30 \mathrm{~mA} \mathrm{~m}{ }^{-2}$. That is, reducing the spike spacing and increasing the spikes could provide a high current density.

Fig. 8 illustrates the effect of spike spacing on discharge current and peak current density of the collection electrode that faces the spikes. The applied voltage was $20.6 \mathrm{kV}$. The discharge

Table 1 Spike spacing and number of spikes

\begin{tabular}{llllll}
\hline Spike spacing $(\mathrm{mm})$ & 25 & 50 & 100 & 150 & 200 \\
Total number of spikes & 111 & 57 & 30 & 21 & 15
\end{tabular}

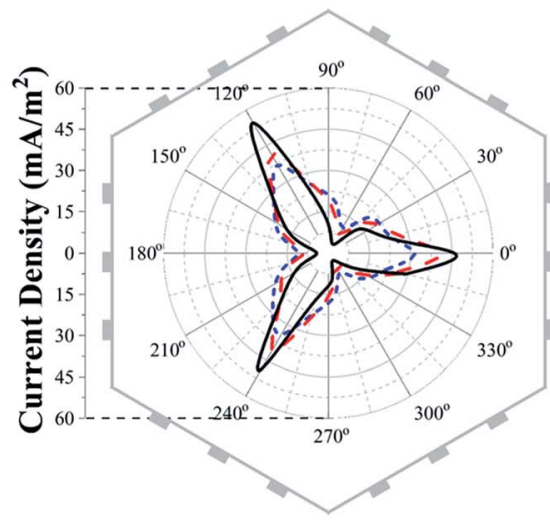

(a)

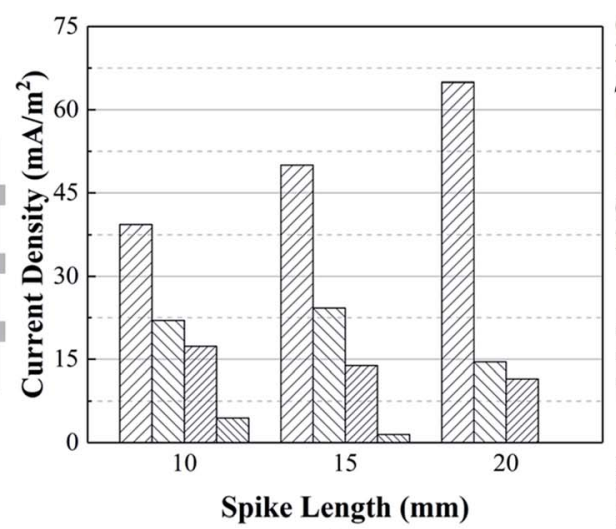

(b)

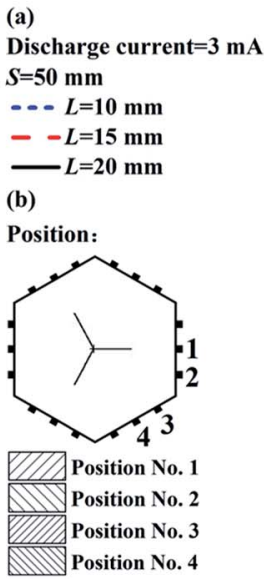

Fig. 6 Current density distribution on the collection electrode of the different spike lengths. 


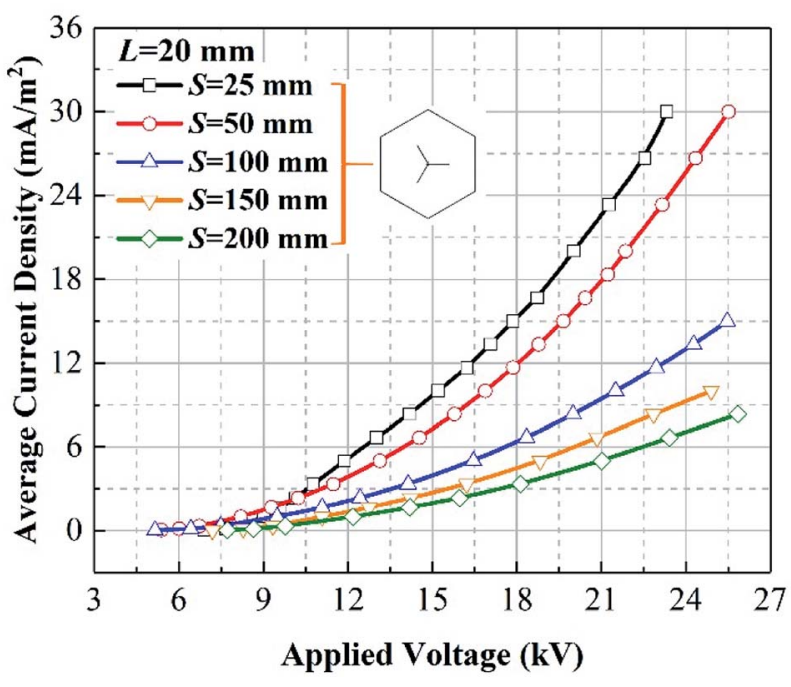

Fig. 7 Effect of spike spacing on the $V-/$ characteristics.

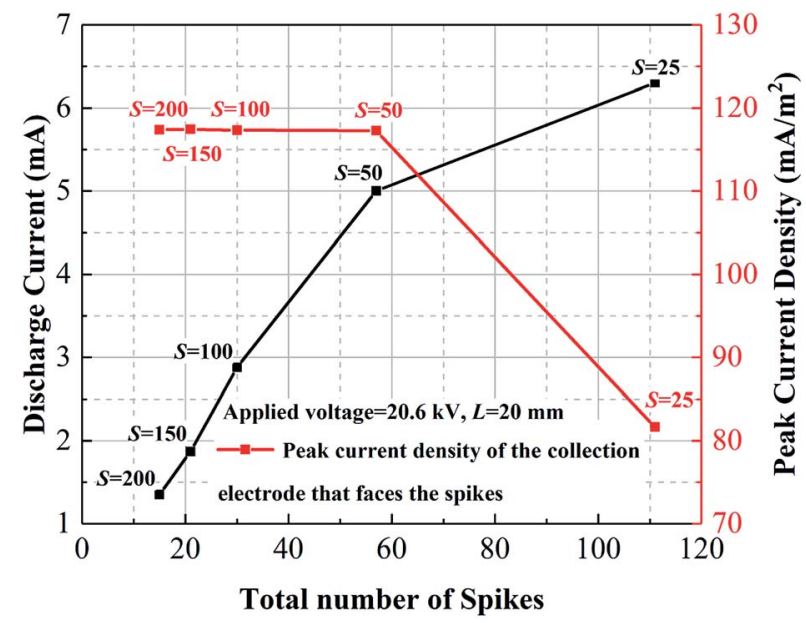

Fig. 8 Effect of spike spacing on discharge current and peak current density of the collection electrode.

current increased at the same voltage with the decrease in spike spacing, but the peak current density slightly changed initially and then decreased. The peak current density had no relation to spike spacing when the latter was larger than $50 \mathrm{~mm}$, and the discharge current and the total number of spikes were positively correlated. The peak current density decreased by $30.1 \%$ at the spike spacing of $25 \mathrm{~mm}$. The result showed that reducing spike spacing can enhance average current density, but the sharp point discharge between spikes was mutually suppressed when spikes were significantly dense. In conclusion, the appropriate spike spacing of the electrode should be selected in accordance with the required discharge current, and $50 \mathrm{~mm}$ was an appropriate spike spacing for this triple-spike electrode.

\subsection{Discharge characteristics affected by the rotation of the discharge electrode}

Triple-spike and sawtooth electrodes exhibited the highest discharge intensities, but the discharge characteristics of the

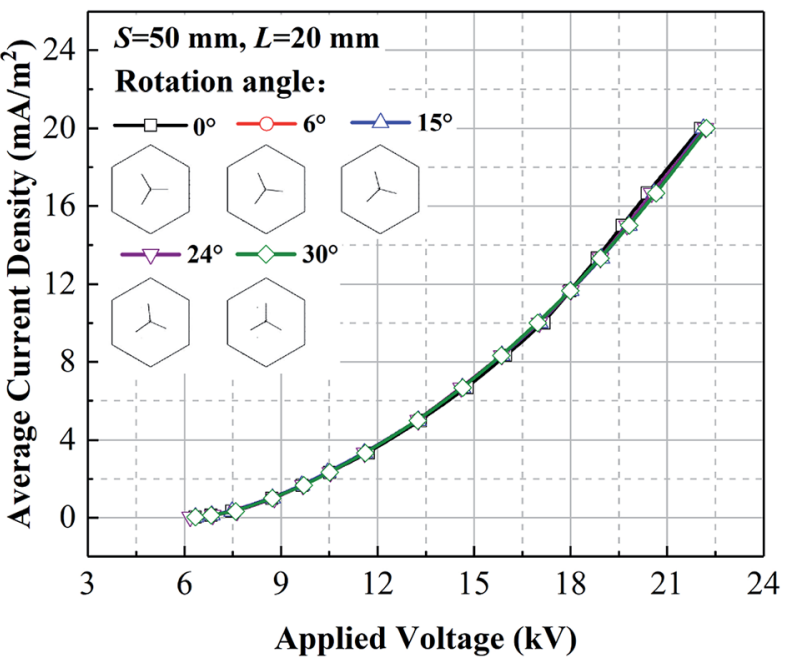

Fig. 9 Effect of rotation angle on the $V-I$ characteristics.

triple-spike electrode were affected by the rotation angle $(\alpha)$ because it was not equidistant between the triple-spike and collection electrodes. $\alpha$ varied from $0^{\circ}$ to $30^{\circ}$ considering the structural symmetry of the triple-spike and honeycomb collection electrodes, and $0^{\circ}$ corresponded to the configuration that spikes were perpendicular to the surface of the collection electrode. Fig. 9 reflects that the rotation of the discharge electrode slightly affected the $V-I$ characteristics.

Fig. 10 illustrates the current density distribution on the collection electrode of different rotation angles. Triple-spike electrode $(S=50 \mathrm{~mm}, L=20 \mathrm{~mm}$ ) were used as the discharge electrode, and the discharge current was $3 \mathrm{~mA}$. The dashed line represented the position of the spikes. The ion diffusion region was changed with the rotation of the discharge electrode from $0^{\circ}$ to $30^{\circ}$ in the wet ESP. The shortest distance between the sharp point and the collection electrode increased, the peak current density decreased, and the position, where the peak current density was located, changed with the rotation. However, the region on the collection electrode where ions could reach increased, and the current density of both sides of the spike gradually increased. The current density distribution on the collection electrode gradually expanded. The average discharge current remained unchanged significantly at the same voltage under two opposite effects on the current density. The current density distribution changed significantly with the slight rotation of the triple-spike electrode. Thus, the accuracy of the electrode installation was particularly important in practical applications.

The current density at four positions with different rotation angles is exhibited in Fig. 11. The trend of the current density change in four positions was different considering the rotation of the discharge electrode from $0^{\circ}$ to $30^{\circ}$. The current density of Position no. 1 decreased by $55.6 \%$ given the rotation of the discharge electrode from $0^{\circ}$ to $30^{\circ}$. The current density of Position no. 2 increased initially and then decreased. The spikes gradually approached Position no. 2 considering the rotation of the discharge electrode, and the current density was largest 


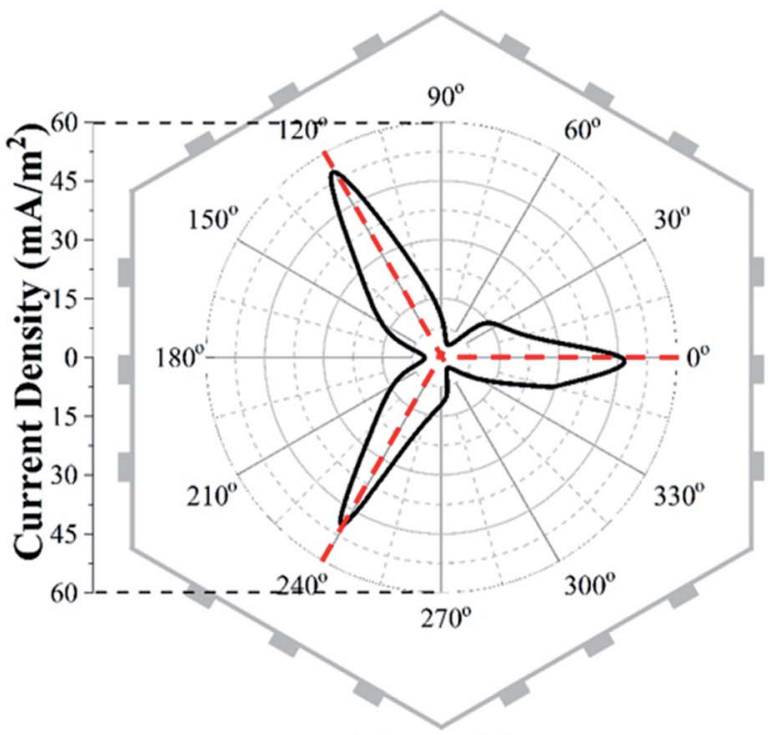

(a) $\alpha=0^{\circ}$

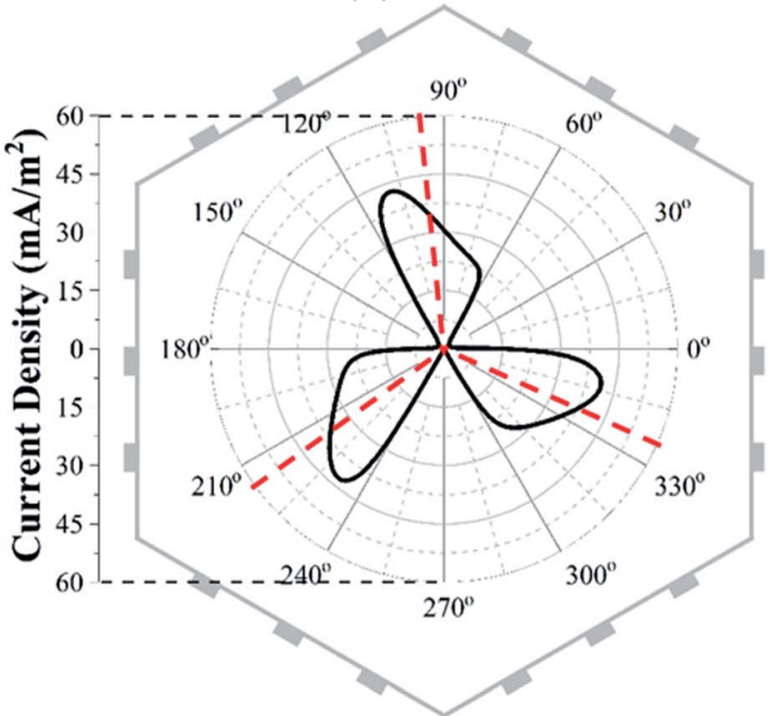

(c) $\alpha=24^{\circ}$

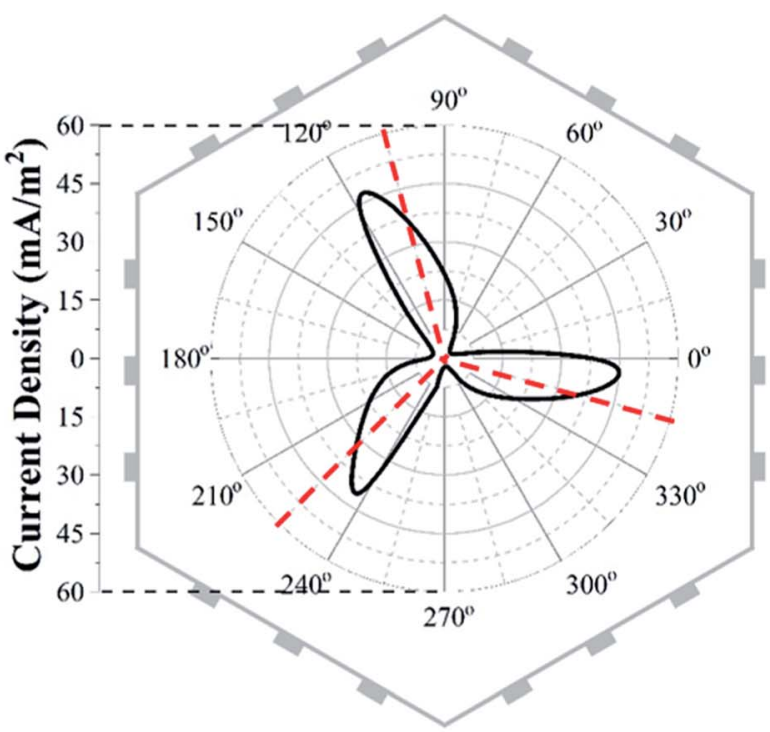

(b) $\alpha=15^{\circ}$

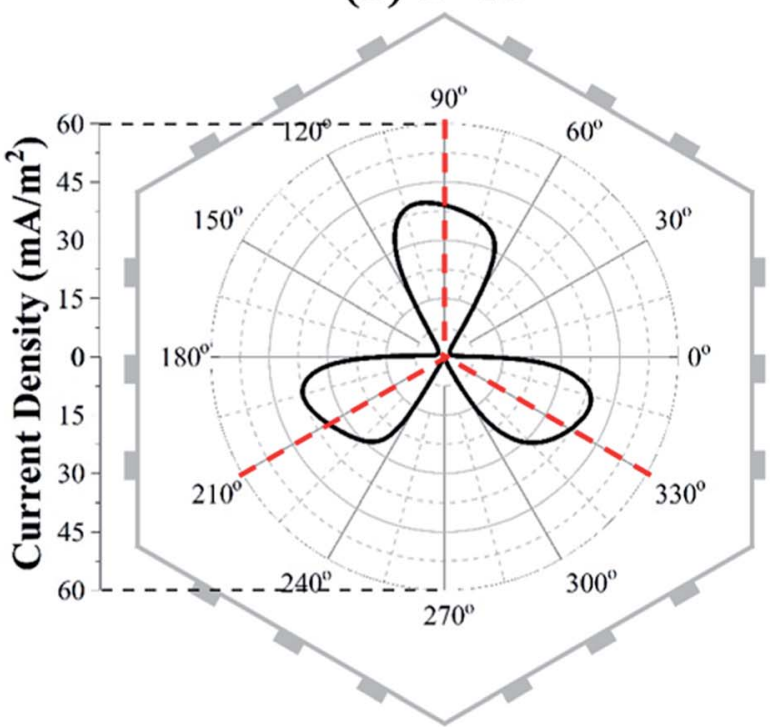

(d) $\alpha=30^{\circ}$

Fig. 10 Current density distribution on the collection electrode of different rotation angles.

when the spike faces Position no. 2. The current density decreased when the spike was distant from Position no. 2. The current density of Position no. 2 increased by $54.1 \%$ compared with the initial state $\left(\alpha=0^{\circ}\right)$. The spike gradually approached Position no. 3 and 4 given the rotation of the discharge electrode, and its current density increased. The current density of Position no. 4 increased from $0 \mathrm{~mA} \mathrm{~m}^{-2}$ to $28.55 \mathrm{~mA} \mathrm{~m}^{-2}$, thereby optimizing the current density distribution effectively.

A statistical analysis was performed on the measured results to analyze the effect of rotation angle on the current density distribution of the collection electrode. Average and standard deviations were calculated using eqn (5) and (6), respectively.

$$
\bar{J}=\frac{1}{n} \sum_{i=1}^{n} J_{i}
$$

$$
\sigma=\sqrt{\frac{1}{n} \sum_{i=1}^{n}\left(J_{i}-\bar{J}\right)^{2}}
$$

where $\bar{J}$ is the average of the current density, $\sigma$ is the standard deviation of the current density, $n$ is the total number of probes, $n$ is set to 171 in this experiment, and $J_{i}$ is the current density of Probe no. $i$.

Fig. 12 presents that the average of the current density changed slightly with the rotation of the discharge electrode from $0^{\circ}$ to $30^{\circ}$. This phenomenon was consistent with the result of the $V-I$ characteristics (Fig. 9). However, the standard deviation of the current density decreased significantly by $20.0 \%$ with the rotation of the electrode when compared with the initial state. That is, the uniformity of the current density distribution increased with the rotation of the discharge electrode, which 


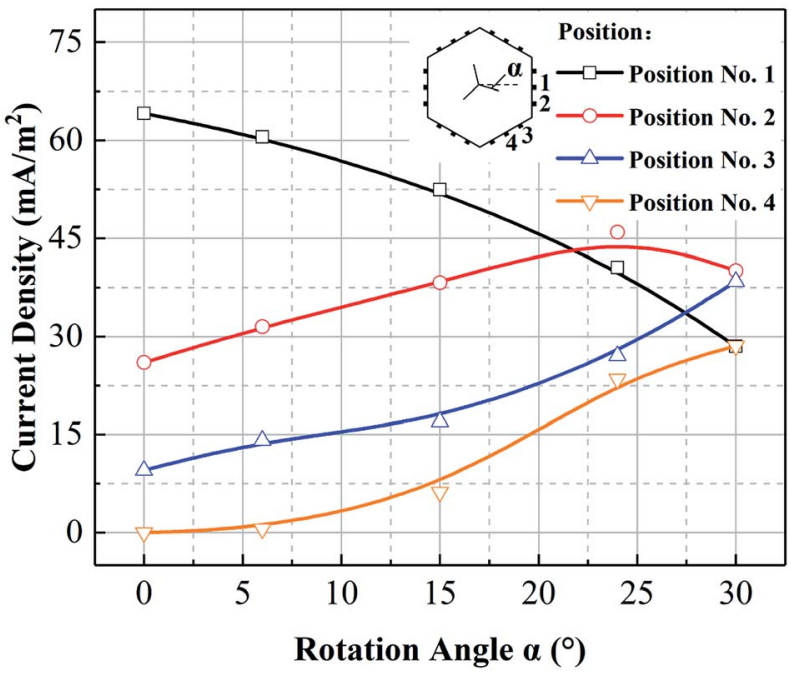

Fig. 11 Comparisons of the current density at four positions with different rotation angles, discharge current $=3 \mathrm{~mA}$.

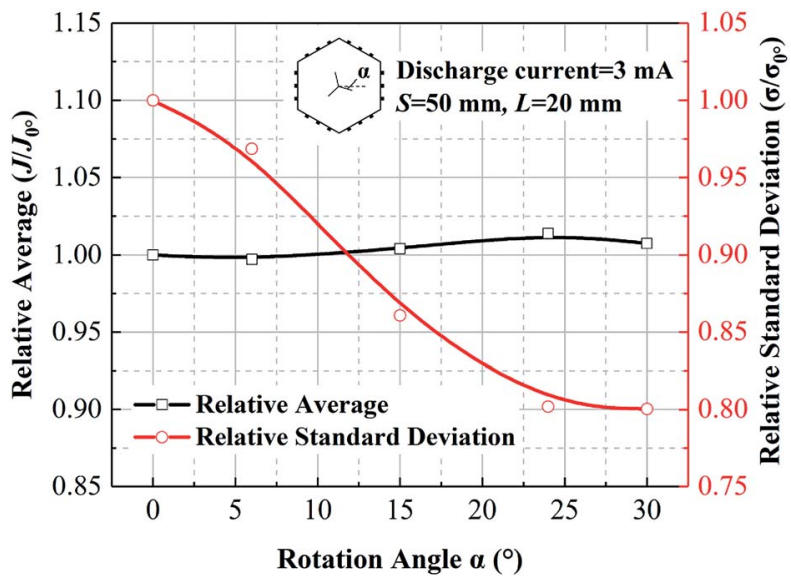

Fig. 12 Average and standard deviations of the current density with different rotation angles.

was consistent with the result of the current density distribution illustrated in Fig. 10. The results showed that the current density distribution of the triple-spike electrode is relatively uniform when the spikes face the edge of the honeycomb collection electrode $\left(\alpha=30^{\circ}\right)$, and $\alpha=30^{\circ}$ is recommended for its overall performance in terms of optimizing the current density distribution.

\subsection{Wet ESP performance for optimized discharge electrode configurations}

In this experiment, the wet ESP performance was evaluated by using the removal efficiency of sulfuric acid aerosol. Triplespike and sawtooth electrodes were used as the discharge electrodes. The environment was controlled at a temperature of $324.15 \pm 1 \mathrm{~K}$ and relative humidity of $100 \%$. The velocity of gas flow and concentration of sulfuric acid aerosol were $2 \mathrm{~m} \mathrm{~s}^{-1}$ and $35.7 \mathrm{mg} \mathrm{m}^{-3}$, correspondingly.

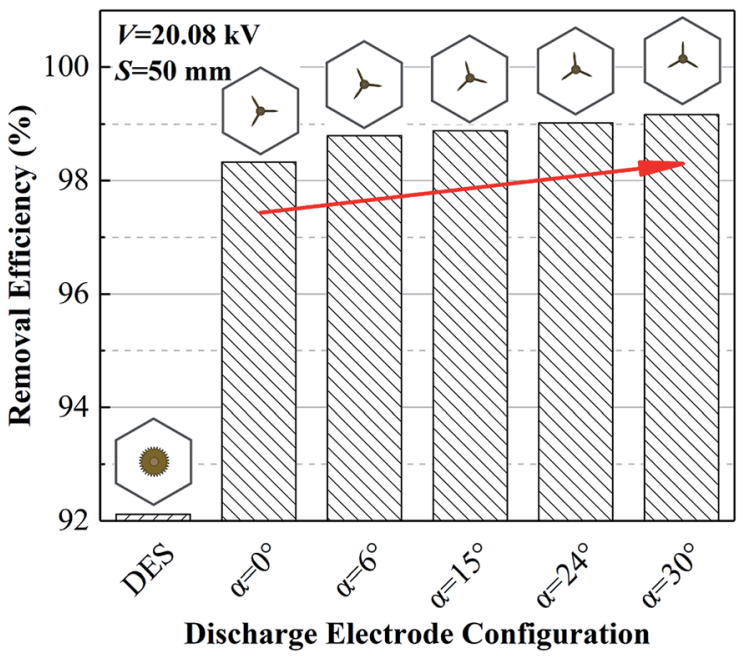

Fig. 13 Effect of discharge electrode configuration on the removal efficiency of sulfuric acid aerosol.

Fig. 13 presents the effect of discharge electrode configuration on the removal efficiency of sulfuric acid aerosol, and the applied voltage was $20.08 \mathrm{kV}$. The removal efficiency exceeded $92 \%$ considering the high average current density of electrode. However, the removal efficiency was $6.3 \%$ lower in the sawtooth electrode, which had the highest average current density, than in the triple-spike electrode $\left(\alpha=0^{\circ}\right)$. This result indicated that the gas flow may be seriously interfered with the discharge of the sawtooth electrode and resulted in the appearance of vortexes near the discharge electrode. Sulfuric acid aerosol cannot be trapped directly by the collection electrode, and the removal efficiency was relatively low in this condition. In terms of the triple-spike electrode, the removal efficiency of sulfuric acid aerosol increased by $0.9 \%$ with the rotation of the discharge electrode from $0^{\circ}$ to $30^{\circ}$, and the highest removal efficiency of sulfuric acid aerosol was $99.2 \%$ at a specific collection area (SCA) of $23.09 \mathrm{~m}^{2}\left(\mathrm{~m}^{3} \mathrm{~s}^{-1}\right)^{-1}$. The removal efficiency of sulfuric acid aerosol could be improved by increasing the uniformity of the discharge current density distribution, and this optimization improved the removal performance of the wet ESP.

\section{Conclusion}

This study compared the effects of electrode geometry and rotation on the negative corona discharge characteristics in a honeycomb wet ESP and experimentally investigated the removal efficiency of sulfuric acid aerosol under optimized discharge electrode configurations. The following conclusions can be drawn from this study:

(1) Average current density was determined on the basis of the discharge electrode type. Triple-spike and sawtooth electrodes demonstrated the highest average current density. The peak current density was $60.5 \%, 133.1 \%$, and $295.8 \%$ higher in the single-spike electrode $(I=3 \mathrm{~mA})$ than in the double-spike, triple-spike, and sawtooth electrodes, correspondingly. The 
current density distribution of the sawtooth electrode was hexagonal and relatively uniform, and all its current density exceeded zero.

(2) The average current density of the triple-spike electrodes increased with the increase in the spike length or the decrease in the spike spacing. The peak current density increased by $62.1 \%$ with the increase in spike length from $10 \mathrm{~mm}$ to $20 \mathrm{~mm}$. The discharge current and the total number of spikes were positively correlated when the spike spacing was larger than 50 $\mathrm{mm}$. The peak current density decreased by $30.1 \%$ when the spike spacing was $25 \mathrm{~mm}$. The reduction in the spike spacing could enhance the average current density, but the sharp point discharge between spikes was mutually suppressed when spikes were significantly dense.

(3) The current density distribution on the collection electrode changed significantly with the slight rotation of the triplespike electrode. Therefore, the accuracy of electrode installation was particularly important in practical applications. Moreover, the electrode configuration in which the spikes face the edge of honeycomb collection electrode $\left(\alpha=30^{\circ}\right)$ is recommended for its overall performance in terms of optimizing the current density distribution.

(4) The removal efficiency was $6.3 \%$ lower in the sawtooth electrode, which had the highest average current density, than in the triple-spike electrode $\left(\alpha=0^{\circ}\right)$. In terms of the triple-spike electrode, the removal efficiency of sulfuric acid aerosol increased by $0.9 \%$ with the rotation of discharge electrode from $0^{\circ}$ to $30^{\circ}$, and the highest removal efficiency of sulfuric acid aerosol was $99.2 \%$ at an SCA of $23.09 \mathrm{~m}^{2}\left(\mathrm{~m}^{3} \mathrm{~s}^{-1}\right)^{-1}$.

\section{Conflicts of interest}

There are no conflicts to declare.

\section{Acknowledgements}

This study was supported by the National Key Research and Development Program of China (Grant No. 2016YFB0600602) and the National Natural Science Foundation of China (Grant No. U1609212 and 51621005).

\section{References}

1 D. Loomis, Y. Grosse, B. Laubysecretan, G. F. El, V. Bouvard, L. Benbrahimtallaa, N. Guha, R. Baan, H. Mattock and K. Straif, The carcinogenicity of outdoor air pollution, Lancet Oncol., 2013, 14(13), 1262-1263.

2 W. H. Organization, ES FS313 Ambient (outdoor) air quality and health. Centro De Prensa, 2014.

3 Y. Landkocz, F. Ledoux, V. Andre, F. Cazier, P. Genevray, D. Dewaele, P. J. Martin, C. Lepers, A. Verdin, L. Courcot, S. Boushina, F. Sichel, M. Gualtieri, P. Shirali, D. Courcot and S. Billet, Fine and ultrafine atmospheric particulate matter at a multi-influenced urban site: physicochemical characterization, mutagenicity and cytotoxicity, Environ. Pollut., 2017, 221, 130-140.
4 J. Li, W. X. Li, C. Bai and Y. Song, Particulate matter-induced epigenetic changes and lung cancer, Clin. Respir. J., 2017, 11(5), 539-546.

5 I. M. Carey, R. W. Atkinson, A. J. Kent, T. van Staa, D. G. Cook and H. R. Anderson, Mortality Associations with Long-Term Exposure to Outdoor Air Pollution in a National English Cohort, Am. J. Respir. Crit. Care Med., 2013, 187(11), 12261233.

6 S. Lu, F. Yi, X. Hao, S. Yu, J. Ren, M. Wu, J. Feng, S. Yonemochi and Q. Wang, Physicochemical properties and ability to generate free radicals of ambient coarse, fine, and ultrafine particles in the atmosphere of Xuanwei, China, an area of high lung cancer incidence, Atmos. Environ., 2014, 97, 519-528.

7 Z. D. Yang, C. H. Zheng, Q. Y. Chang, Y. Wan, Y. Wang, $X$. Gao and K. F. Cen, Fine particle migration and collection in a wet electrostatic precipitator, J. Air Waste Manage. Assoc., 2017, 67(4), 498-506.

8 C. Wang, X. W. Liu, D. Li, J. P. Si, B. Zhao and M. H. Xu, Measurement of particulate matter and trace elements from a coal-fired power plant with electrostatic precipitators equipped the low temperature economizer, Proc. Combust. Inst., 2015, 35, 2793-2800.

9 Z. D. Yang, C. H. Zheng, X. F. Zhang, Q. Y. Chang, W. G. Weng, Y. Wang and X. Gao, Highly efficient removal of sulfuric acid aerosol by a combined wet electrostatic precipitator, RSC Adv., 2018, 8(1), 59-66.

10 Y. S. Xu, X. W. Liu, J. Cui, D. Chen, M. H. Xu, S. W. Pan, K. Zhang and X. P. Gao, Field Measurements on the Emission and Removal of PM2.5 from Coal-Fired Power Stations: 4. PM Removal Performance of Wet Electrostatic Precipitators, Energy Fuels, 2016, 30(9), 7465-7473.

11 S. Sinanis, A. Wix, L. Ana and K. Schaber, Characterization of sulphuric acid and ammonium sulphate aerosols in wet flue gas cleaning processes, Chem. Eng. Process., 2008, 47(1), 2230 .

12 Z. Yang, C. Zheng, X. Zhang, H. Zhou, A. A. Silva, C. Liu, B. Snyder, Y. Wang and X. Gao, Challenge of $\mathrm{SO}_{3}$ removal by wet electrostatic precipitator under simulated flue gas with high $\mathrm{SO}_{3}$ concentration, Fuel, 2018, 217, 597-604.

13 A. Mizuno, Electrostatic precipitation, IEEE Trans. Dielectr. Electr. Insul., 2000, 7(5), 615-624.

14 M. R. Beltran, Wet ESP for the Collection of Submicron Particles, Mist and Air Toxics, Springer Berlin Heidelberg, 2009, pp. 499-506.

15 R. C. Staehle, R. J. Triscori, K. Sampath, F. L. Smidth, G. Ross and R. Cothron, Wet Electrostatic Precipitators for High Efficiency Control of Fine Particulates and Sulfuric Acid Mist, 2003.

16 Z. D. Yang, C. H. Zheng, X. F. Zhang, C. J. Li, Y. Wang, W. G. Weng and X. Gao, Sulfuric Acid Aerosol Formation and Collection by Corona Discharge in a Wet Electrostatic Precipitator, Energy Fuels, 2017, 31(8), 8400-8406.

17 U. Khaled and A. Z. Eldein, Experimental study of $V-I$ characteristics of wire-plate electrostatic precipitators under clean air conditions, J. Electrost., 2013, 71(3), 228-234. 
18 Q. Y. Lu, Z. D. Yang, C. H. Zheng, X. Li, C. Zhao, X. Xu, X. Gao, Z. Y. Luo, M. J. Ni and K. F. Cen, Numerical simulation on the fine particle charging and transport behaviors in a wire-plate electrostatic precipitator, $A d v$. Powder Technol., 2016, 27(5), 1905-1911.

19 M. Jedrusik and A. Swierczok, The correlation between corona current distribution and collection of fine particles in a laboratory-scale electrostatic precipitator, J. Electrost., 2013, 71(3), 199-203.

20 A. Jaworek, A. Krupa and T. Czech, Modern electrostatic devices and methods for exhaust gas cleaning: a brief review, J. Electrost., 2007, 65(3), 133-155.

21 M. Jedrusik, J. B. Gajewski and A. J. Swierczok, Effect of the particle diameter and corona electrode geometry on the particle migration velocity in electrostatic precipitators, $J$. Electrost., 2001, 51, 245-251.

22 M. Ali, K. Alam, Y. Al-Majali and M. Kennedy, Novel hybrid composite discharge electrode for electrostatic precipitator, J. Air Waste Manage. Assoc., 2017, 67(9), 1036-1045.

23 X. Wang, J. Chang, C. Xu, P. Wang, L. Cui and C. Ma, Electrical characteristics of electrostatic precipitator with a wet membrane-based collecting electrode, J. Electrost., 2016, 80, 85-94.

24 P. Yan, C. Zheng, W. Zhu, X. Xu, X. Gao, Z. Luo, M. Ni and K. Cen, An experimental study on the effects of temperature and pressure on negative corona discharge in high-temperature ESPs, Appl. Energy, 2016, 164(9), 28-35.

25 N. Farnoosh, K. Adamiak and G. S. P. Castle, Threedimensional analysis of electrohydrodynamic flow in a spiked electrode-plate electrostatic precipitator, $J$. Electrost., 2011, 69(5), 419-428.

26 X. Xu, C. Zheng, P. Yan, W. Zhu, Y. Wang, X. Gao, Z. Luo, M. Ni and K. Cen, Effect of electrode configuration on particle collection in a high-temperature electrostatic precipitator, Sep. Purif. Technol., 2016, 166, 157-163.

27 P. Yan, C. Zheng, G. Xiao, X. Xu, X. Gao, Z. Luo and K. Cen, Characteristics of negative DC corona discharge in a wire- plate configuration at high temperatures, Sep. Purif. Technol., 2015, 139, 5-13.

28 X. Xu, X. Gao, P. Yan, W. Zhu, C. Zheng, Y. Wang, Z. Luo and K. Cen, Particle migration and collection in a hightemperature electrostatic precipitator, Sep. Purif. Technol., 2015, 143, 184-191.

29 Q. Y. Chang, C. H. Zheng, Z. D. Yang, M. X. Fang, X. Gao, Z. Y. Luo and K. F. Cen, Electric agglomeration modes of coal-fired fly-ash particles with water droplet humidification, Fuel, 2017, 200, 134-145.

30 X. Wang, J. Chang, C. Xu, J. Zhang, P. Wang and C. Ma, Collection and charging characteristics of particles in an electrostatic precipitator with a wet membrane collecting electrode, J. Electrost., 2016, 83, 28-34.

31 N. Farnoosh, K. Adamiak and G. S. P. Castle, Numerical Calculations of Submicron Particle Removal in a SpikePlate Electrostatic Precipitator, IEEE Trans. Dielectr. Electr. Insul., 2011, 18(5), 1439-1452.

32 H. Fujishima, Y. Morita, M. Okubo and T. Yamamoto, Numerical simulation of three-dimensional electrohydrodynamics of spiked-electrode electrostatic precipitators, IEEE Trans. Dielectr. Electr. Insul., 2006, 13(1), 160-167.

33 B. Y. Guo, J. Guo and A. B. Yu, Simulation of the electric field in wire-plate type electrostatic precipitators, J. Electrost., 2014, 72(4), 301-310.

34 X. Wang, M. Ni, G. Xiao, J. Zhang, X. Gao and K. Cen, An analytical method for DC negative corona discharge in a wire-cylinder device at high temperatures, J. Electrost., 2014, 72(4), 270-284.

35 G. Xiao, X. Wang, G. Yang, M. Ni, X. Gao and K. Cen, An experimental investigation of electrostatic precipitation in a wire-cylinder configuration at high temperatures, Powder Technol., 2015, 269, 166-177.

36 Z. Gu, X. Xi, J. Yang and J. Xu, Properties of RE-W cathode and its application in electrostatic precipitation for high temperature gas clean-up, Fuel, 2012, 95(1), 648-654. 